\title{
Confinement Effects on Polymer Dynamics: Thermo-Responsive Behaviours of Hydroxypropyl Cellulose Polymers in Phospholipid-Coated Droplets (Water-in-Oil Emulsion)
}

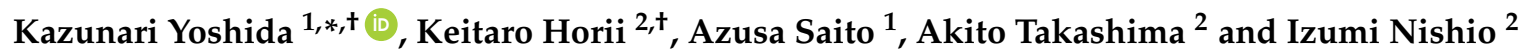 \\ 1 Department of Mechanical Systems Engineering, Graduate School of Science and Engineering, \\ Yamagata University, 4-3-16, Jonan, Yonezawa 992-8510, Yamagata, Japan; azusasaito@yz.yamagata-u.ac.jp \\ 2 Department of Physics and Mathematics, College of Science and Engineering, Aoyama Gakuin University, \\ 5-10-1 Fuchinobe, Chuo-ku, Sagamihara 252-5258, Kanagawa, Japan; horii.k13@nishio-lab.net (K.H.); \\ takashima.a.aa@phys.aoyama.ac.jp (A.T.); izumi24058@yahoo.co.jp (I.N.) \\ * Correspondence: k-yoshida@yz.yamagata-u.ac.jp; Tel.: +81-238-26-3218 \\ + These authors contributed equally to this work.
}

Received: 7 November 2017 ; Accepted: 1 December 2017 ; Published: 6 December 2017

\begin{abstract}
In order to construct the artificial cells and to understand the physicochemical properties of living cells, it is important to clarify the cell-sized confinement effect on the behaviours of bio-inspired polymers. We report the dynamic behaviours of aqueous hydroxypropyl cellulose (HPC) solution coated with phospholipids in oil (water-in-oil droplets, W/O droplets), accompanied by an increase in the temperature. We directly observed the beginning of phase separation of HPC solution using a fluorescence microscope and confirmed the dependence of such phenomena on droplet size. The results indicate that the start time of phase separation is decreased with an increase in droplet size. The experimental results suggest that the confinement situation accelerates the phase separation of aqueous HPC solutions.
\end{abstract}

Keywords: dynamics; thermo-responsive polymer; water-in-oil droplet (emulsion); artificial cell model

\section{Introduction}

Recently, a physicochemical approach to artificial cells has been studied with a view to its applications in medical science, in addition to the research of embryonic stem (ES) $[1,2]$ and induced pluripotent stem (iPS) [3] cells. It is beneficial to utilize the preparation methods of the liposome [4-6] and the water-in-oil (W/O) droplet coated by phospholipid [7,8] for constructing an artificial cell because such systems are representative simple model systems of living cell and/or cell membranes and can be prepared easily. It is known that living cells possess the various types of polymers with high concentration, and biochemical reaction and folding of protein might be influenced by crowding [9]. Hence, it is very important to clarify the physicochemical effect of confinement on behaviours of polymers.

In the 1970s, it was revealed that coil-globule transition of a single polymer chain was induced by various stimuli such as change of solvent composition [10]. Helix-coil transitions of biopolymers has been also studied for many years [11-13]. Phase separation of polymers has been reported, in addition to the phase transition $[14,15]$. It is speculated that such fundamental properties and behaviours of polymers are also influenced by crowding.

There are many studies for clarifying the effect of confinement on behaviours of polymers to date. For instance, the group of Dimova and Lipowsky has revealed the contact angle between polymer-rich and water-rich phases enclosed by lipid bilayer vesicles [16-19]. Several resemble studies are also 
reported for clarifying the interfacial phenomena in confined phase-separated polymer solution [20,21]. Kato et al. reported that gene expression is significantly influenced by confinement, utilizing $\mathrm{W} / \mathrm{O}$ droplet coated with phospholipids [22]. Further studies of confinement effect on dynamic behaviours of polymers is required for establishing artificial cells.

Hydroxypropyl cellulose (HPC) is one of the representative biocompatible polymers since its main component is a multi-sugar chain, and the living cell possesses various kinds of sugar derivative molecules such as glycolipids [23]. The phase behaviours of aqueous HPC solution have been widely studied utilizing spectroscopic methods [24-26]. The results of previous studies show that HPC solutions exhibit a lower critical solution temperature (LCST) at ca. $42{ }^{\circ} \mathrm{C}[25,26]$. Furthermore, it has been shown that volume phase transition of HPC hydrogels is also significantly correlated with the LCST [27-29]. However, dynamic phase behaviour of HPC solution enclosed by phospholipids is still ambiguous.

In this study, we report the relationship between droplet size and dynamic behaviour of aqueous HPC solution. The results of the experiments indicate that the starting time of phase separation of HPC solution is significantly correlated with droplet size.

\section{Materials and Methods}

\subsection{Materials}

Mineral oil was obtained from Nacalai Tesque, Inc. (Kyoto, Japan). Such mineral oil has been widely used in the studies of $\mathrm{W} / \mathrm{O}$ droplets $[8,21]$. Ultra-pure water was obtained using a WT101UV Autopure system of Yamato Scientific Co., Ltd. (Tokyo, Japan). Electrical resistivity of the obtained ultra-pure water was more than $18 \mathrm{M} \Omega \cdot \mathrm{cm}$ at $25{ }^{\circ} \mathrm{C}$. HPC $(2.0-2.9 \mathrm{mPa} \cdot \mathrm{s})$, calcein (fluorescent dye, $\lambda_{\mathrm{ex}} / \lambda_{\mathrm{em}}=495 \mathrm{~nm} / 515 \mathrm{~nm}$ ) and chloroform were obtained from Wako Pure Chemical Industries, Ltd. (Osaka, Japan). 1,2-dipalmitoyl-sn-glycero-3-phosphocholine (DPPC) was obtained from Avanti Polar Lipids, Inc. (Birmingham, AL, USA). Rhodamine B 1,2-dihexadecanoyl-sn-glycero-3-phosphoethanolamine, triethylammonium salt (rhodamine DHPE, fluorescent lipid, $\lambda_{\mathrm{ex}} / \lambda_{\mathrm{em}}=560 \mathrm{~nm} / 580 \mathrm{~nm}$ ), was obtained from Invitrogen (Carlsbad, CA, USA).

\subsection{Preparation of HPC Solution Droplets}

First, DPPC ( $2 \mathrm{mM})$ and small amount of rhodamine DHPE were dissolved in chloroform $(105 \mu \mathrm{L})$, and the lipid solution was poured into a small test tube. Then, the solvent of the solution was removed to form a thin dry lipid film under vacuum conditions. A volume of $400 \mu \mathrm{L}$ of mineral oil was poured into the test tube with lipid film, and then, the tube was sonicated for $90 \mathrm{~min}$ at $60^{\circ} \mathrm{C}$ to disperse the lipids all over the oil. Finally, $40 \mathrm{wt} \%$ of aqueous HPC solution $(40 \mu \mathrm{L})$ was added to the lipid and oil solution, and emulsification was performed via sonication for $10 \mathrm{~min}$ and pipetting. The final concentration of rhodamine DHPE and calcein were $0.01 \mathrm{mM}$ (calculated) in the mineral oil and $0.1 \mathrm{mM}$ (measured) in the aqueous HPC solution, respectively. The HPC solution droplets are stable in several days, and the droplets were observed within a day from that those ware made. The droplet sized are up to $130 \mu \mathrm{m}$ in diameters, and the deviation of size is shown below (Results and Discussion).

\subsection{Microscopic Observation under Temperature Control}

A volume of $20 \mu \mathrm{L}$ of sample suspension including HPC solution droplets was put between two glass coverslips and sealed with vacuum grease. The sample cell was put on the hand-made temperature-control system (deviation $<0.1^{\circ} \mathrm{C}$, see Figure 1a). We used three thermistors; the two were used for temperature monitoring, and the other was for feedback in order to control the plate temperature. The temperature of copper plate was controlled through a proportional integral (PI) circuit and a Peltier device (deviation $<0.1{ }^{\circ} \mathrm{C}$ ). The measured temperature was defined as an averaged value using two thermistors. The thickness of the sample suspension is less than $1 \mathrm{~mm}$. The temperature control system is shown in Figure 1a. We observed the HPC solution droplets on the 
temperature-control system using a fluorescence microscope, BX40 (Olympus, Tokyo, Japan) equipped with a $\times 40$ objective lens (Olympus, Japan), a digital camera DP73 (Olympus, Japan) and a Hg lamp. We observed the lipid monolayer (rhodamine DHPE) using a Olympus WIG filter set $\left(\lambda_{\mathrm{ex}} / \lambda_{\mathrm{em}}=\right.$ $520-550 \mathrm{~nm} /$ above $580 \mathrm{~nm}$ ) and the HPC solution (inside the droplets, calcein) using a Olympus NIBA filter set $\left(\lambda_{\mathrm{ex}} / \lambda_{\mathrm{em}}=470-490 \mathrm{~nm} / 510-550 \mathrm{~nm}\right)$. The dynamics of the HPC solution droplet was observed when we changed the temperature from $10{ }^{\circ} \mathrm{C}$ to $65^{\circ} \mathrm{C}$ (see Figure $1 \mathrm{~b}$ ). The videos were recorded at $7 \mathrm{fps}$.

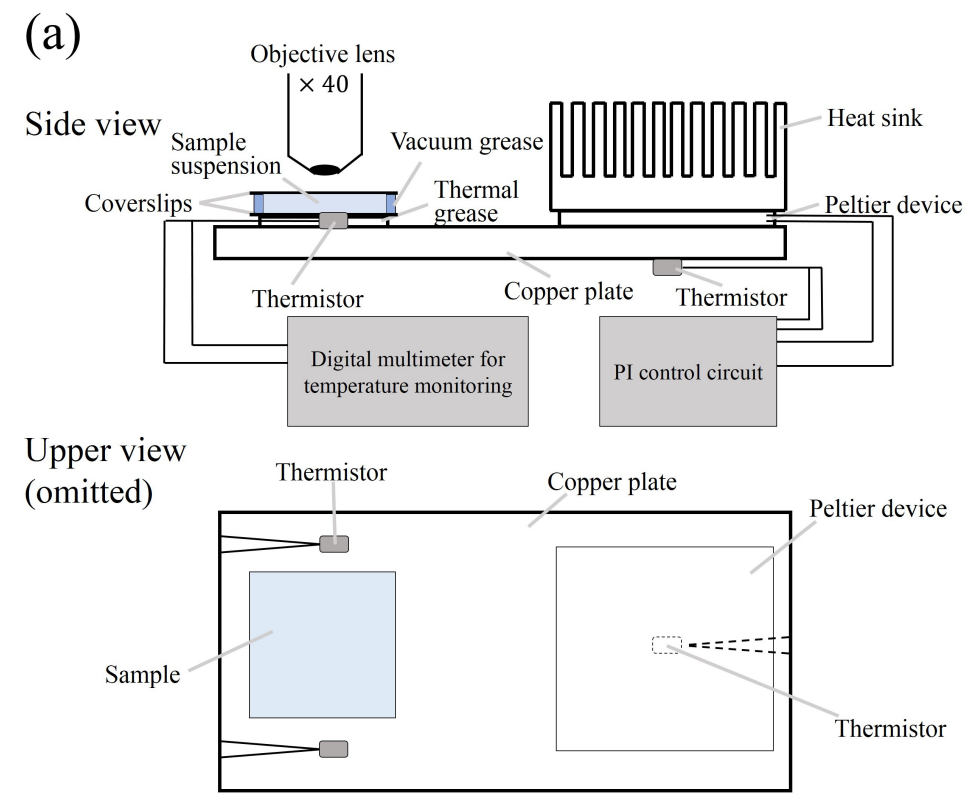

(b)

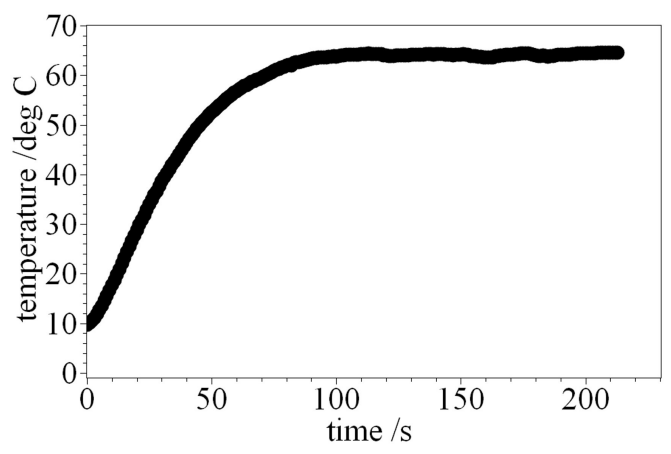

Figure 1. (a) Schematic illustration of temperature-control system. We used three thermistors; the two were for temperature monitoring, and the other was for feedback in order to control the plate temperature. The temperature of the copper plate was controlled through a proportional integral (PI) circuit and a Peltier device (deviation $<0.1^{\circ} \mathrm{C}$ ). The measured temperature was defined as an averaged value using two thermistors. Thickness of the sample suspension is within $1 \mathrm{~mm}$; (b) The thermal profile was measured by thermistors in the case of the situation from $10^{\circ} \mathrm{C}$ to $65^{\circ} \mathrm{C}$. The temperature reached above ca. $42{ }^{\circ} \mathrm{C}$ within $50 \mathrm{~s}$ from the beginning of heating.

\section{Results and Discussion}

Figure 2a shows the schematic illustration of HPC solution droplet which is water-in-oil (W/O) type droplet. Such droplets can be formed through the procedure as shown in previous section. We confirmed the such mesoscopic structure using a optical microscopy (see Figure $2 b, c$ ). Figure $2 b$ indicates that rhodamine DHPE is mainly localized in the rim of the droplets while calcein is 
mainly localized inside of the droplets. These facts suggest that the HPC droplets floating in the oil phase are coated with DPPC lipids since rhodamine DHPE and calcein are mainly localized in phospholipid-rich [7] and water-rich [30] regions, respectively.

(a)

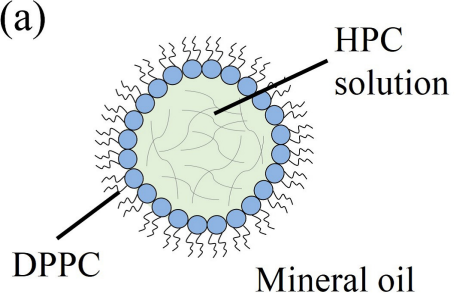

(b)

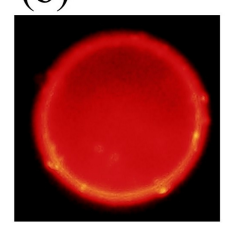

(c)

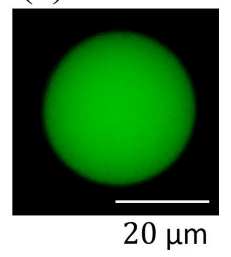

Figure 2. (a) Schematic illustration of HPC solution droplet (W/O type dorplet); (b) Microscopic images in the case of observation of dyes rhodamine DHPE, $\lambda_{\mathrm{em}}=580 \mathrm{~nm}$ (red), corresponding to place of lipids; (c) Microscopic images in the case of observation of dyes calcein, $\lambda_{\mathrm{em}}=515 \mathrm{~nm}$ (green), corresponding to place of water molecules inside the droplet, and in this photograph, the water molecules (also the HPC) are uniformly dispersed.

Figure 3 shows typical time-lapse images of HPC droplet, observed by fluorescence microscopy when we changed the temperature from $10{ }^{\circ} \mathrm{C}$ to $65^{\circ} \mathrm{C}$. With increasing elapsed time, the dot-like pattern appeared inside the droplet. This indicates that phase separation was caused by increase in the temperature since the HPC has LCST in the aqueous solution $[25,26]$. The bright region corresponds to water-rich phase while the dark region (inside droplet) corresponds to HPC-rich phase since calcein can be dissolved in aqueous solution [30]. This result shows that the phase separation progressed from the interface to the centre of the droplet. This phenomenon suggests that the head groups of lipids influenced the phase dynamics of HPC solution.

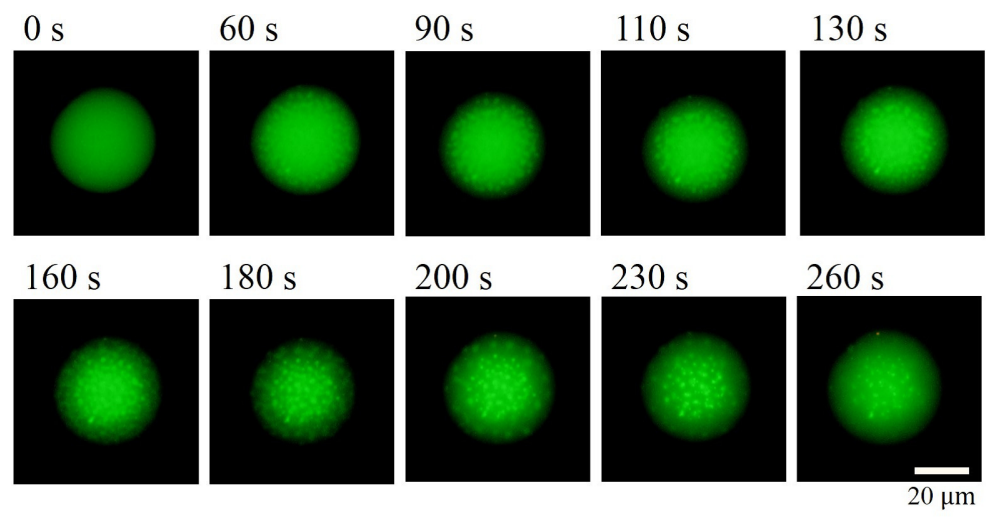

Figure 3. Typical microscopic images of hydroxypropyl cellulose (HPC) droplet with elapsed time when we changed the temperature from $10{ }^{\circ} \mathrm{C}$ to $65^{\circ} \mathrm{C}$. The dot-like phase-separation pattern appeared with an increase in temperature. The phase separation progressed from the interface to the centre of the droplet. The bright region corresponds to the water-rich phase, while the dark region inside the droplet corresponds to the HPC-rich phase.

We investigated the Feret's diameters $\left(d_{\mathrm{F}}\right)$, which are the maximum caliper of HPC droplet (droplet size), derived from cross section images using a software ImageJ [31]. Figure 4a shows the histogram of the Feret's diameters $(n=33)$. The result of statistical analysis shows that HPC droplet size is $34 \pm 27 \mu \mathrm{m}$ (median $\pm \mathrm{SD}$ ). We are able to prepare the HPC droplets with up to $130 \mu \mathrm{m}$ using this emulsification procedure. 
Next, we investigated the size dependency of the start time of phase separation on droplet sizes/interaction ratio between HPC and DPPC $(R)$. As shown above, phase separation is induced by an increase in temperature, and the elapsed time when the temperature begins to be increased from $10{ }^{\circ} \mathrm{C}$. This corresponds to easiness of phase separation (velocity of phase separation dynamics). Furthermore, we also defined the interaction ratio between HPC and DPPC as follows:

$$
R=\frac{\pi d_{\mathrm{F}}}{\pi d_{\mathrm{F}}^{2} / 4 \pi}=\frac{4 \pi}{d_{\mathrm{F}}}
$$

which is the perimeter to area of cross section ratio of HPC droplets. Figure $4 \mathrm{~b}$ shows the relationship between start time of phase separation and droplet size/interaction ratio between HPC and DPPC. The start time of the phase separation is increased with an increase in droplet size. This result indicates that a longer time is required for the phase separation in the case of larger HPC droplets. In contrast, the interaction ratio is decreased with an increase in start time of phase separation, which indicates that a shorter time is required for the phase separation in the case of higher interaction between HPC and lipids. These facts suggest that the confinement situation accelerates the phase separation of aqueous HPC solutions although the effect of size dependency of thermal conductivity is ignorable. For the relationship between interaction ratio $R$ and start time of phase separation (see Figure 4), it is considered that the lipid head groups influenced the dynamic phase behaviours of aqueous HPC solution. In other words, there is a possibility that DPPC lipids coating the HPC droplets have an ability to accelerate the phase separation. This is in good agreement with the fact that the phase separation progressed from the interface of the droplet (see Figure 3). To confirm such a possibility, further experiments are required. However, to the best of our knowledge, this is the first report showing the effect of droplet size/lipids on dynamic phase behaviour of aqueous HPC solutions.

(a)

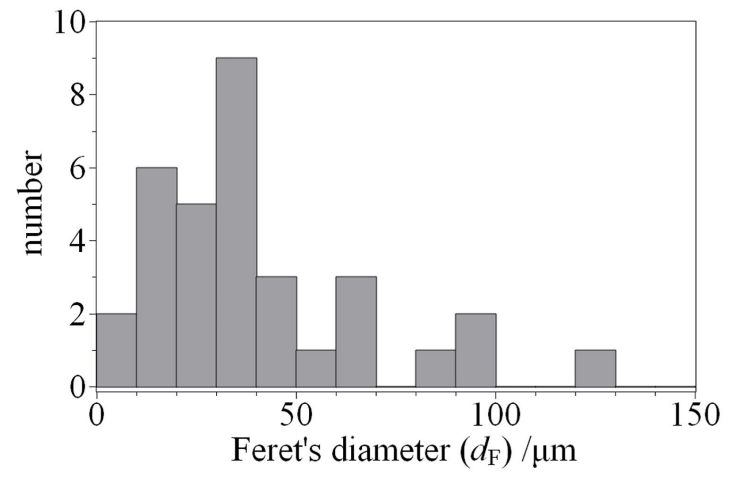

(b)

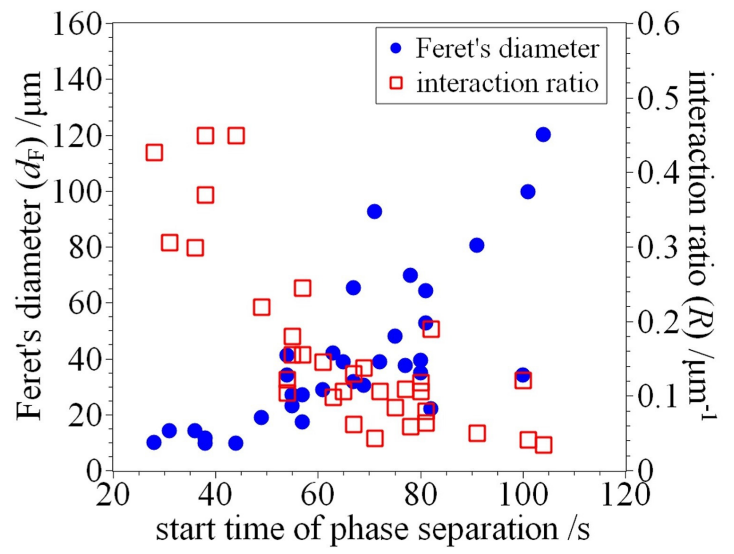

Figure 4. (a) Histogram plot of Feret's diameter $\left(d_{\mathrm{F}}\right)$ of HPC droplets (droplet size, $n=33$ ). The value of Feret's diameter is $34 \pm 27 \mu \mathrm{m}$ (median $\pm \mathrm{SD}$ ); (b) Relationship between start time of phase separation and $d_{\mathrm{F}}$ of HPC droplets/interaction ratio between HPC and 1,2-dipalmitoyl-snglycero-3-phosphocholine (DPPC) $(R)$.

For the present results of relationship between $R$ and start time of phase separation, it is considered that the lipid head groups influence the movement of HPC polymers and water molecules. In this study, the DPPC, which has a zwitterion in the head group, has been used for coating the droplet. The HPC polymers hardly bind to the lipids coated interface since the zwitterion, including bio-inspired materials, have been widely investigated for anti-fouling materials [32]. Hence, further experiments using lipids possessing other types of head groups, such as 
1,2-dipalmitoyl-sn-glycero-3-phospho-(1'-rac-glycerol) (DPPG), are required in order to clarify the effects of interface on the interactions between HPC, lipids and water molecules.

Previously, Kato et al. reported that gene expression rate (protein production rate) is proportional to $1 / r$, where $r$ is the radius of the confinement droplet radius. This means that protein expression is accelerated in a smaller confinement space [22]. We consider that the acceleration of this biochemical gene expression and phase separation of the polymer is very important information to construct the artificial cells and to understand the living cells. Hence, such investigations may lead to further understanding of the optimum size of an artificial cell for constructing the various types of cells.

Kurokawa et al. reported that the artificial cytoskeleton made with deoxyribonucleic acid (DNA) stabilizes the artificial cells [33]. In addition, Terasawa et al. reported that the budding of lipid bilayer vesicles (similar to cell division) is induced by depletion effect by polymers inside the vesicles [34]. We have to combine the knowledges obtained from previous studies and the present study in order to construct the artificial cells and to understand the physicochemical properties of living cells.

\section{Conclusions}

In summary, we investigated the dynamic phase behaviours of aqueous hydroxypropyl cellulose (HPC) solution coated with phospholipids in oil (water-in-oil droplets, W/O droplets), accompanied with an increase in the temperature. The experimental results indicate that the start time of phase separation of HPC droplets is strongly correlated with droplet size/interaction ratio between HPC and DPPC. It is considered that the confinement situation accelerates the phase separation of aqueous HPC solutions. In addition, the importance of lipid head group coating droplets was also suggested. Hence, in order to understand the detailed effect of lipids on polymer dynamics, further experiments are required using different types of lipids. The finding of this study and the results of further experiments may lead to a detailed understanding of the optimum size of artificial cells and then to a new range of studies of artificial cell and detailed understanding of the physicochemical properties of living cells.

Acknowledgments: The authors would like to thank Tsutomu Hamada at Japan Advanced Institute of Science and Technologies for his significant advises.

Author Contributions: Kazunari Yoshida designed the experiments and wrote the manuscript with revisions by Akito Takashima and Izumi Nishio. Keitaro Horii performed the experiments with supports by Kazunari Yoshida and Azusa Saito.

Conflicts of Interest: The authors declare no conflict of interest.

\section{Abbreviations}

The following abbreviations are used in this manuscript:

HPC hydroxypropyl cellulose

W/O droplets water-in-oil droplets

DPPC 1,2-dipalmitoyl-sn-glycero-3-phosphocholine

DPPG 1,2-dipalmitoyl-sn-glycero-3-phospho-(1'-rac-glycerol)

\section{References}

1. Evans, M.J.; Kaufman, M.H. Establishment in culture of pluripotential cells from mouse embryos. Nature 1981, 292, 154-156.

2. Martin, G.R. Isolation of a pluripotent cell line from early mouse embryos cultured in medium conditioned by teratocarcinoma stem cells. Proc. Natl. Acad. Sci. USA 1981, 78, 7634-7638.

3. Takahashi, K.; Yamanaka, S. Induction of Pluripotent Stem Cells from Mouse Embryonic and Adult Fibroblast Cultures by Defined Factors. Cell 2006, 126, 663-676.

4. Yoshida, K.; Fujii, Y.; Nishio, I. Deformation of Lipid Membranes Containing Photoresponsive Molecules in Response to Ultraviolet Light. J. Phys. Chem. B 2014, 118, 4115-4121.

5. Yoshida, K.; Horii, K.; Fujii, Y.; Nishio, I. Real-Time Observation of Liposome Bursting Induced by Acetonitrile. ChemPhysChem 2014, 15, 2909-2912. 
6. Yoshida, K.; Takashima, A.; Nishio, I. Effect of Dibucaine Hydrochloride on Raft-Like Lipid Domains in Model Membrane Systems. Med. Chem. Commun. 2015, 6, 1444-1451.

7. Hamada, T.; Morita, M.; Kishimoto, Y.; Komatsu, Y.; Vestergaard, M.; Takagi, M. Biomimetic Microdroplet Membrane Interface: Detection of the Lateral Localization of Amyloid Beta Peptides. J. Phys. Chem. Lett. 2010, 1, 170-173.

8. Hamada, T.; Fujimoto, R.; Shimobayashi, S.F.; Ichikawa, M.; Takagi, M. Molecular behavior of DNA in a cell-sized compartment coated by lipids. Phys. Rev. E 2015, 91, 062717.

9. Ellis, R.J.; Minton, A.P. Cell biology: Join the crowd. Nature 2003, 425, 27-28.

10. Nishio, I.; Sun, S.T.; Swislow, G.; Tanaka, T. First observation of the coil-globule transition in a single polymer chain. Nature 1979, 281, 208-209.

11. Tanaka, T.; Soda, K.; Wada, A. Dynamical aspects of helix-coil transitions in polypeptides. I. J. Chem. Phys. 1973, 58, 5707-5715.

12. Tanaka, T.; Wada, A.; Suzuki, M. Dynamical aspects of helix-coil transitions in biopolymers. II. J. Chem. Phys. 1973, 59, 3799-3810.

13. Bonduelle, C.; Makni, F.; Severac, L.; Piedra-Arroni, E.; Serpentini, C.L.; Lecommandoux, S.; Pratviel, G. Smart metallopoly(L-glutamic acid) polymers: Reversible helix-to-coil transition at neutral pH. RSC Adv. 2016, 6, 84694-84697.

14. Yanagisawa, M.; Yamashita, Y.; atsu Mukai, S.; Annaka, M.; Tokita, M. Phase separation in binary polymer solution: Gelatin/Poly(ethylene glycol) system. J. Mol. Liq. 2014, 200, 2-6.

15. Yamashita, Y.; Yanagisawa, M.; Tokita, M. Sol-gel transition and phase separation in ternary system of gelatin-water-poly(ethylene glycol) oligomer. J. Mol. Liq. 2014, 200, 47-51.

16. Kusumaatmaja, H.; Li, Y.; Dimova, R.; Lipowsky, R. Intrinsic Contact Angle of Aqueous Phases at Membranes and Vesicles. Phys. Rev. Lett. 2009, 103, 238103.

17. Li, Y.; Kusumaatmaja, H.; Lipowsky, R.; Dimova, R. Wetting-Induced Budding of Vesicles in Contact with Several Aqueous Phases. J. Phys. Chem. B 2012, 116, 1819-1823.

18. Dimova, R.; Lipowsky, R. Lipid membranes in contact with aqueous phases of polymer solutions. Soft Matter 2012, 8, 6409-6415.

19. Dimova, R.; Lipowsky, R. Giant Vesicles Exposed to Aqueous Two-Phase Systems: Membrane Wetting, Budding Processes, and Spontaneous Tubulation. Adv. Mater. Interfaces 2017, 4, 1600451.

20. Keating, C.D. Aqueous phase separation as a possible route to compartmentalization of biological molecules. Acc. Chem. Res. 2012, 45, 2114-2124.

21. Yanagisawa, M.; Nigorikawa, S.; Sakaue, T.; Fujiwara, K.; Tokita, M. Multiple patterns of polymer gels in microspheres due to the interplay among phase separation, wetting, and gelation. Proc. Natl. Acad. Sci. USA 2014, 111, 15894-15899.

22. Kato, A.; Yanagisawa, M.; Sato, Y.T.; Fujiwara, K.; Yoshikawa, K. Cell-sized confinement in microspheres accelerates the reaction of gene expression. Sci. Rep. 2012, 2, 283.

23. Alberts, B.; Johnson, A.; Lewis, J.; Morgan, D.; Raff, M.; Roberts, K.; Walter, P. Molecular Biology of the Cell, 6th ed.; Garland Science: New York, NY, USA, 2014.

24. Fortin, S.; Charlet, G. Phase diagram of aqueous solutions of (hydroxypropyl)cellulose. Macromolecules 1989, 22, 2286-2292.

25. Winnik, F.M.; Tamai, N.; Yonezawa, J.; Nishimura, Y.; Yamazaki, I. Temperature-induced phase transition of pyrene-labeled (hydroxypropyl) cellulose in water: Picosecond fluorescence studies. J. Phys. Chem. 1992, 96, 1967-1972.

26. Guido, S. Phase behavior of aqueous solutions of hydroxypropyl cellulose. Macromolecules 1995, 28, 4530-4539.

27. Kabra, B.G.; Gehrke, S.H.; Spontak, R.J. Microporous, Responsive Hydroxypropyl Cellulose Gels. 1. Synthesis and Microstructure. Macromolecules 1998, 31, 2166-2173.

28. Adrados, B.; Galaev, I.; Nilsson, K.; Mattiasson, B. Size exclusion behavior of hydroxypropylcellulose beads with temperature-dependent porosity. J. Chromatogr. A 2001, 930, 73-78.

29. Xia, X.; Tang, S.; Lu, X.; Hu, Z. Formation and Volume Phase Transition of Hydroxypropyl Cellulose Microgels in Salt Solution. Macromolecules 2003, 36, 3695-3698.

30. Hamada, T.; Kishimoto, Y.; Nagasaki, T.; Takagi, M. Lateral phase separation in tense membranes. Soft Matter 2011, 7, 9061-9068. 
31. Schneider, C.A.; Rasband, W.S.; Eliceiri, K.W. NIH Image to ImageJ: 25 Years of Image Analysis. Nat. Methods 2012, 9, 671-675.

32. Damodaran, V.B.; Murthy, N.S. Bio-inspired strategies for designing antifouling biomaterials. Biomater. Res. 2016, 20, 18.

33. Kurokawa, C.; Fujiwara, K.; Morita, M.; Kawamata, I.; Kawagishi, Y.; Sakai, A.; Murayama, Y.; Nomura, S.M.; Murata, S.; Takinoue, M.; et al. DNA cytoskeleton for stabilizing artificial cells. Proc. Natl. Acad. Sci. USA 2017, 114, 7228-7233.

34. Terasawa, H.; Nishimura, K.; Suzuki, H.; Matsuura, T.; Yomo, T. Coupling of the fusion and budding of giant phospholipid vesicles containing macromolecules. Proc. Natl. Acad. Sci. USA 2012, 109, 5942-5947.

(C) 2017 by the authors. Licensee MDPI, Basel, Switzerland. This article is an open access article distributed under the terms and conditions of the Creative Commons Attribution (CC BY) license (http://creativecommons.org/licenses/by/4.0/). 